\title{
Another Look at Square Roots (and Other Less Common Operations) in Fields of Even Characteristic
}

\author{
Roberto Maria Avanzi \\ Faculty of Mathematics and Horst Görtz Institute for IT-Security \\ Ruhr University Bochum, Germany \\ Roberto.Avanzi@ruhr-uni-bochum.de
}

\begin{abstract}
We discuss a family of irreducible polynomials that can be used to speed up square root extraction in fields of characteristic two. They generalize trinomials discussed by Fong et al. 20. We call such polynomials square root friendly.

The main application is to point halving methods for elliptic curves (and to a lesser extent also divisor halving methods for hyperelliptic curves and pairing computations).

We note the existence of square root friendly trinomials of a given degree when we already know that an irreducible trinomial of the same degree exists, and formulate a conjecture on the degrees of the terms of square root friendly polynomials. Following similar results by Bluher, we also give a partial result that goes in the direction of the conjecture.

We also discuss how to improve the speed of solving quadratic equations. The increase in the time required to perform modular reduction is marginal and does not affect performance adversely. Estimates confirm that the new polynomials mantain their promises. Point halving gets a speed-up of $20 \%$ and scalar multiplication is improved by at least $11 \%$.
\end{abstract}

Keywords: Binary fields, Polynomial basis, Square root extraction, Trace computation, Quadratic equations, Point halving.

\section{Introduction}

The main topic of this paper is square root extraction in binary fields, even though also other operations are considered.

In [20] it is shown that the extraction of square roots can be accelerated if a suitable irreducible trinomial is used to define the field.

Let $p(X)$ be the irreducible polynomial of degree $d$ used to define the extension field $\mathbb{F}_{2^{d}} / \mathbb{F}_{2}$. If the polynomial in $X$ representing the square root of the image $x$ of $X$ in $\mathbb{F}_{2^{d}}$ has low weight and/or degree, then general square roots can be extracted in $\mathbb{F}_{2^{d}}$ efficiently. In this case we call $p(X)$ square root friendly. (This definition will be made more precise later.)

We give here sufficient conditions for an irreducible polynomial of odd degree $d$ to yield a low weight $\sqrt{x}$. In particular, we give examples of pentanomials

C. Adams, A. Miri, and M. Wiener (Eds.): SAC 2007, LNCS 4876, pp. 138 154, 2007.

(C) Springer-Verlag Berlin Heidelberg 2007 
and heptanomials, but in at least one case, that of $\mathbb{F}_{2^{233}}$, that can be defined by trinomials, we show how one can perform square root computations even faster than in 20$]$.

As the motivation comes from elliptic curve cryptography, in particular from point halving based methods for scalar multiplication, in Section 2 we recall point and divisor halving. In Section 3 we introduce square root computations and our new sufficient conditions. Polynomials for several useful (and used in practice) binary fields are presented in Section 4. together with a result about the existence of square root friendly trinomials, a conjecture about the degrees of the non-leading terms of square root friendly polynomials, and a theorem that supports the conjecture itself.

Finally, we move to implementation issues in Section 5. Not only square root extraction is implemented $(\S 5.1)$, but also the computation of traces over $\mathbb{F}_{2}$ $(\S 5.2)$ and solving quadratic equations in $\mathbb{F}_{2^{d}}(\oint 5.3)$. These routines are benchmarked showing gains, whereas multiplication and squaring either experience a negligible slowdown or even a minimal speedup. The costs of various elliptic curve group operations and scalar multiplication algorithms using different reduction polynomials for the definition field are also given. The discussion of these results $(\$ 5.4)$ also provides the conclusion to the paper. In particular, we prove that a performance gain of around $20 \%$ can be expected for the point halving alone, with a speed increase in excess of $11 \%$ for scalar multiplication.

Space constraints forced us to omit most proofs. These, as well as further results, will be given in the full version of the paper.

\section{Point and Divisor Halving}

Let $E$ be an elliptic curve defined over $\mathbb{F}_{2^{d}}$ by a Weierstrass equation

$$
E: Y^{2}+X Y=X^{3}+a X^{2}+b
$$

with $a, b \in \mathbb{F}_{2^{d}}$ and having a subgroup $G \leq E\left(\mathbb{F}_{2^{d}}\right)$ of large prime order.

Since computing the double of any given point $P$ is the most common operation in a scalar multiplication performed by double-and-add methods, an important direction of research consists in optimizing doubling formulæ (for surveys on scalar multiplication methods and elliptic curve operations see, for example [8, Chs. 9 and 13] or [21, Ch. 3]).

Point halving [22 27], on the other hand, consists in computing a point $R$ whose double is $P$, i.e. such that $2 R=P$. Being the inverse operation of the doubling, it is an automorphism of $G$. Therefore, given a point $P \in G$, there is a unique $R \in G$ such that $2 R=P$.

In order to perform this operation one needs to solve a quadratic equation of the form $\lambda^{2}+\lambda+\alpha=0$ for $\lambda$, extract a square root, perform two multiplications

\footnotetext{
${ }^{1}$ Scott [28] considers searching the polynomials until the one giving the optimal reduction and square root routines is found in terms of the amount of logic and shift operations.
} 
and some additions. We refer the reader to [22 26/27|20] for details, including the usage of halving in place of doubling in scalar multiplication algorithms. Furthermore, there are two points $R_{1}$ and $R_{2}$ on the curve with $2 R_{1}=2 R_{2}=P$, such that $R_{1}-R_{2}$ is the unique point of order 2 of the curve. To determine which one is in $G$, an additional check involving a trace computation is required. Knudsen 22] and Schroeppel [2627] show how to perform all these operations. According to the analysis in [20], halving is about two times faster than doubling.

Birkner 14 has devised a divisor halving formula for genus two curves based on the doubling formulae by Lange and Stevens [24]. Birkner and Thériault [15] have dealt with genus three divisors. The performance of all known halving formulæ depends (to a variable degree) on the performance of square root extraction. Further uses of point halving to speed up scalar multiplication on elliptic Koblitz Curves [23] are found in [7,9] and [10].

\section{Square Root Extraction and Defining Polynomials}

\subsection{Background}

Let $p(X)$ be an irreducible polynomial of odd degree $d$, and the field $\mathbb{F}_{2^{d}}$ be constructed as the quotient ring $\mathbb{F}_{2}[X] /(p(X))$. Let us call $x$ the image of $X$ in $\mathbb{F}_{2^{d}}$. We consider here polynomial basis representation because we are solely concerned with software applications.

Whereas with a normal basis 4 a square root computation is just a shift of the bits internal representation of the field element by one position, matters are more complicated with polynomial bases. In fact, even the cost of a squaring becomes no longer negligible. If $\alpha=\sum_{i=0}^{d-1} a_{i} x^{i}$ then $\alpha^{2}=\sum_{i=0}^{d-1} a_{i} x^{2 i}$ which, as a polynomial in $x$, has degree no longer necessarily bounded by $d$, and modular reduction (modulo $p(X))$ is necessary. Its cost is very low, but cannot be completely ignored.

Things are more complicated for square roots. Even though squaring just consists in "spacing" the bits of the original element with zeros, the bits of a generic field element cannot be just "compressed". The classic method for computing $\sqrt{\alpha}$ is based on Fermat's little theorem $\alpha^{2^{d}}=\alpha$, hence $\sqrt{\alpha}=\alpha^{2^{d-1}}$. This requires $d-1$ squarings. In general, the cost of this operation is that of several field multiplications.

A more efficient method stems from the observation that $\sqrt{\alpha}$ can be expressed in terms of $\zeta:=\sqrt{x}$. If

$$
\alpha=\sum_{i=0}^{d-1} a_{i} x^{i}
$$

we separate the even exponents from the odd exponents

$$
\alpha=\sum_{i=0}^{\frac{d-1}{2}} a_{2 i} x^{2 i}+\sum_{i=0}^{\frac{d-3}{2}} a_{2 i+1} x^{2 i+1}=\left(\alpha_{\text {even }}\right)^{2}+x \cdot\left(\alpha_{\text {odd }}\right)^{2}
$$


where

$$
\alpha_{\text {even }}=\sum_{i=0}^{\frac{d-1}{2}} a_{2 i} x^{i} \quad \text { and } \quad \alpha_{\text {odd }}=\sum_{i=0}^{\frac{d-3}{2}} a_{2 i+1} x^{i}
$$

and, since square root in a field of even characteristic is a linear operation:

$$
\sqrt{\alpha}=\alpha_{\text {even }}+\zeta \cdot \alpha_{\text {odd }}
$$

Therefore, once $\zeta$ has been computed on a per-field basis, the computation of a generic square root is reduced to "bits extraction and packing", a "rectangular" multiplication of a degree $\leq d-1$ polynomial $\zeta$ with a polynomial $\sum_{i=0}^{(d-3) / 2} a_{2 i+1} x^{i}$ of degree $\leq(d-3) / 2$ in $x$, and a modular reduction. Intuitively, the cost should approach a half of the cost of a field multiplication and this is confirmed by the analysis in [20 22].

\subsection{Square-Root Friendly Polynomials}

The speed of square root computation depends on the efficiency of the multiplication of a generic polynomial of degree $\leq(d-1) / 2$ by $\zeta=\sqrt{x}$. If $\zeta$ is very sparse, for example of weight two or four (i.e. it has just two or four nonzero terms), then this product can be computed by a few shift and XOR operations. In [20] two types of trinomials have been shown that allow this. The kind that interests us is

$$
p(X)=X^{d}+X^{m}+1
$$

with $m$ odd. Then $x=x^{d+1}+x^{m+1}$ with $d+1$ and $m+1$ even, and

$$
\zeta=x^{(d+1) / 2}+x^{(m+1) / 2},
$$

and $p(X)$ is square root friendly. In fact, this idea is much more general.

Assume the irreducible polynomial $p(X)$ defining $\mathbb{F}_{2^{d}}$ over $\mathbb{F}_{2}$ has form

$$
p(X)=X \cdot \mathcal{U}(X)^{2}+1
$$

where $\mathcal{U}$ is a polynomial of degree $(d-1) / 2$ and even weight $w$ (hence $p(X)$ has weight $w+1)$. Then, $\zeta$ has a very simple form in $\mathbb{F}_{2^{d}}$ : from $x^{2} \cdot \mathcal{U}(x)^{2}+x=0$ we obtain

$$
\zeta=x \cdot \mathcal{U}(x),
$$

and $\zeta$ is represented by a polynomial of degree $1+\frac{d-1}{2}=\frac{d+1}{2}$ and weight $w$. Furthermore, note that the polynomial product

$$
\zeta \cdot \sum_{i=0}^{\frac{d-3}{2}} a_{2 i+1} x^{i}
$$

has degree bounded by $\frac{d+1}{2}+\frac{d-3}{2}=d-1$, therefore no polynomial reduction is required if a square root is computed by formula (10).

Hence, irreducible polynomials of form (2) are square root friendly. 
Note that the low degree of $\zeta$ not only guarantees that no modular reduction is necessary, but puts also a bound on the complexity of the multiplication by $\zeta$ (even though its sparseness has an even bigger influence). It is therefore interesting to ask whether there are irreducible polynomials $P(X)$ that lead to a $\zeta$ of even lower degree. This cannot happen, and requiring the degree of the polynomial representing $\zeta$ to be at most $(d+1) / 2$ in fact almost characterizes the polynomials (2).

Theorem 1. Let the field extension $\mathbb{F}_{2^{d}} / \mathbb{F}_{2}$ be defined by an irreducible polynomial $P(X)$ of odd degree $d$, and let $x$ be a zero of $P(X)$ in $\mathbb{F}_{2^{d}}$. Suppose that $\zeta:=\sqrt{x}$ is a polynomial of degree at most $(d+1) / 2$ in $x$.

Then the degree of $\zeta$ is exactly $(d+1) / 2$ and either

(i) $P(X)=1+X \cdot \mathcal{U}(X)^{2}$ and $\zeta=x \cdot \mathcal{U}(x)$ for some $\mathcal{U}(X) \in \mathbb{F}_{2}[X]$ of degree exactly $(d-1) / 2$; or

(ii) $P(X)=1+(X+1) \cdot X^{2} \cdot \mathcal{W}(X)^{2}$ and $\zeta=1+(x+1) \cdot x \cdot \mathcal{W}(x)$ for some $\mathcal{W}(X) \in \mathbb{F}_{2}[X]$ of degree exactly $(d-3) / 2$.

Definition 1. An irreducible polynomial of form (2) is called a special square root friendly (SSRF) polynomial of type I.

An irreducible polynomial

$$
P(X)=1+(X+1) \cdot X^{2} \cdot \mathcal{W}(X)^{2}
$$

for some $\mathcal{W}(X) \in \mathbb{F}_{2}[X]$ of degree exactly $(d-3) / 2$ is called a special square root friendly polynomial of type II.

We do not know whether there are irreducible polynomials which are not trinomials, not of the forms listed in Theorem 1, and for which $\sqrt{x}$ has small weight. Even for trinomials $X^{d}+X^{m}+1$ with even $m$ one has to check on a case by case basis, but examples are known 20.

Still, square root friendly polynomials abound. For example $X^{163}+X^{57}+$ $X^{49}+X^{29}+1$ is irreducible, and the corresponding $\zeta$ has weight 4 . On the other hand, the standard NIST polynomial 25] $X^{163}+X^{7}+X^{6}+X^{3}+1$ defines a $\zeta$ of weight 79 .

Remark 1. Changing polynomial is easy without introducing incompatibilities in cryptographic applications: we just change the base used for representation of the field elements before and after the bulk of the computation. The cost is comparable to a polynomial basis multiplication, and the conversion routines require each a matrix that occupies $O\left(d^{2}\right)$ bits of storage (cf. 19] where the particular base change is to and from a normal basis representation, but the results are the same). Therefore this overhead is essentially negligible with respect to the full cost of a scalar multiplication, that is in the order of magnitude of hundreds to thousands of field multiplications (see for example $\S 5.3$ of [5]).

Remark 2. The cost of a square root extraction implemented by using the sparse version of $\zeta$ offered by the above polynomials can be roughly estimated using, 
for example, already published results. For example in [20], Example 3.12, the NIST-recommended trinomial

$$
p(X)=X^{233}+X^{74}+1
$$

for the finite field $\mathbb{F}_{2^{233}}$ is used. Even though the term $X^{74}$ does not have an odd exponent, $\zeta$ has a sparse representation

$$
\zeta=\left(x^{32}+x^{117}+x^{191}\right)\left(x^{37}+1\right) .
$$

By this, finding a root via equation (1) requires roughly $1 / 8$ of the time of a field multiplication. As we shall show in the next section we can choose

$$
p(X)=X^{233}+X^{159}+1
$$

and in this case

$$
\zeta=x^{117}+x^{80} .
$$

It is clear that an even smaller amount of shift operations and XOR operations are required to multiply by $\zeta$. Furthermore, as already remarked, there is no need to perform a reduction modulo $p(X)$ while with non-SSRF polynomials this is almost always necessary, even with the polynomial (4). Implementation results show the cost of a square root to be less than $9 \%$ of that of a multiplication. See $\S 5.4$ for precise results.

Remark 3. Similar formulæ for cube root computations are found in [1] - their results are easily partially generalised to any odd characteristic.

Remark 4. Type I SSRF polynomials enjoy another very useful property. In [2] it is proved that the only trace-one element in the polynomial basis defined by these polynomials is 1 . Therefore the trace of an element $\sum_{i=0}^{d-1} a_{i} x^{i}$ is just $a_{0}$. This is very important, for instance, for point halving. It is especially fortunate that the same family of polynomials makes both square roots and trace computations faster.

Type II SSRF polynomials do not enjoy this property, however they are not relevant anyway for most applications: They were also investigated in [2], because all element of the polynomial basis, except at most for 1 , have trace one, but the connection to the minimality of the degree of $\sqrt{x}$ is new and surprising.

\section{Existence and Degrees of the Terms}

Square root friendly polynomials are easy to find. For example, a simple MAGMA 17. script determines all 713 (special) square root friendly pentanomials of degree 163 in less than one minute.

In Table 1 we list square root friendly polynomials of several degrees. The degrees have been taken from the NIST list of recommended binary curves and from the extension degrees used in $[12$. All these extension degrees are interesting 
Table 1. Some special square root friendly trinomials and pentanomials

\begin{tabular}{|c|c|c|c|}
\hline 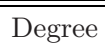 & Irreducible tri/pentanomial & $\zeta=\sqrt{x}$ & Standard? \\
\hline 47 & $X^{47}+X^{5}+1$ & $x^{24}+x^{3}$ & Yes \\
\hline 53 & $X^{53}+X^{19}+X^{17}+X^{15}+1$ & $x^{27}+x^{10}+x^{9}+x^{8}$ & No \\
\hline 59 & $X^{59}+X^{21}+X^{17}+X^{15}+1$ & $x^{30}+x^{11}+x^{9}+x^{8}$ & No \\
\hline 67 & $X^{67}+X^{25}+X^{17}+X^{5}+1$ & $x^{34}+x^{13}+x^{9}+x^{3}$ & No \\
\hline 71 & $X^{71}+X^{9}+1$ & $x^{36}+x^{5}$ & No \\
\hline 73 & $X^{73}+X^{25}+1$ & $x^{37}+x^{13}$ & Yes \\
\hline 79 & $X^{79}+X^{9}+1$ & $x^{40}+x^{5}$ & Yes \\
\hline 83 & $X^{83}+X^{29}+X^{25}+X^{3}+1$ & $x^{42}+x^{15}+x^{13}+x^{2}$ & No \\
\hline 89 & $X^{89}+X^{51}+1$ & $x^{45}+x^{26}$ & No \\
\hline 97 & $X^{97}+X^{33}+1$ & $x^{49}+x^{17}$ & No \\
\hline 101 & $X^{101}+X^{35}+X^{31}+X^{3}+1$ & $x^{51}+x^{18}+x^{16}+x^{2}$ & No \\
\hline 107 & $X^{107}+X^{37}+X^{33}+X^{23}+1$ & $x^{54}+x^{19}+x^{17}+x^{12}$ & No \\
\hline 109 & $X^{109}+X^{43}+X^{41}+X^{23}+1$ & $x^{55}+x^{22}+x^{21}+x^{12}$ & No \\
\hline 127 & $X^{127}+X+1$ & $x^{64}+x$ & Yes \\
\hline 131 & $X^{131}+X^{45}+X^{41}+X^{9}+1$ & $x^{66}+x^{23}+x^{21}+x^{5}$ & No \\
\hline 137 & $X^{137}+X^{21}+1$ & $x^{69}+x^{11}$ & Yes \\
\hline 139 & $X^{139}+X^{53}+X^{33}+X^{25}+1$ & $x^{70}+x^{27}+x^{17}+x^{13}$ & No \\
\hline 149 & $X^{149}+X^{51}+X^{47}+X^{9}+1$ & $x^{75}+x^{26}+x^{24}+x^{5}$ & No \\
\hline 157 & $X^{157}+X^{55}+X^{47}+X^{11}+1$ & $x^{79}+x^{28}+x^{24}+x^{6}$ & No \\
\hline 163 & $X^{163}+X^{57}+X^{49}+X^{29}+1$ & $x^{82}+x^{29}+x^{25}+x^{15}$ & No \\
\hline 179 & $X^{179}+X^{61}+X^{57}+X^{41}+1$ & $x^{90}+x^{31}+x^{29}+x^{21}$ & No \\
\hline 199 & $X^{199}+X^{67}+1$ & $x^{100}+x^{34}$ & No \\
\hline 211 & $X^{211}+X^{73}+X^{69}+X^{35}+1$ & $x^{106}+x^{37}+x^{35}+x^{18}$ & No \\
\hline 233 & $X^{233}+X^{159}+1$ & $x^{117}+x^{80}$ & No \\
\hline 239 & $X^{239}+X^{81}+1$ & $x^{120}+x^{41}$ & No \\
\hline 251 & $X^{251}+X^{89}+X^{81}+X^{3}+1$ & $x^{126}+x^{45}+x^{41}+x^{2}$ & No \\
\hline 269 & $X^{269}+X^{91}+X^{87}+X^{61}+1$ & $x^{135}+x^{46}+x^{44}+x^{31}$ & No \\
\hline 283 & $X^{283}+X^{97}+X^{89}+X^{87}+1$ & $x^{142}+x^{49}+x^{45}+x^{44}$ & No \\
\hline 409 & $X^{409}+X^{87}+1$ & $x^{205}+x^{44}$ & Yes \\
\hline 571 & $X^{571}+X^{193}+X^{185}+X^{5}+1$ & $x^{286}+x^{97}+x^{93}+x^{3}$ & No \\
\hline
\end{tabular}

because they are either used in standards for elliptic curve cryptography or they represent good choices for extension degrees for defining hyperelliptic curve for cryptographic applications.

When no trinomial is available, a pentanomial is listed. We always report the polynomial with least degree sediment (the sediment of an univariate is obtained by removing the leading term). Only in a handful of cases is the square root friendly polynomial with least degree sediment the same as the standard one, i.e. the irreducible polynomial with least degree sediment but without the restriction on being square root friendly.

We first note that if an irreducible trinomial of a given degree exists, then we can always find one which is square root friendly. The following result is in fact well known, and we state it only for completeness' sake.

Theorem 2. Let d be an odd positive integer. If an irreducible trinomial $p(X)=$ $X^{d}+X^{m}+1$ over $\mathbb{F}_{2}$ of degree $d$ exists, then $p(X)$ can be chosen of form (2), i.e. where the exponent $m$ of the middle term is odd. 
Existence results for pentanomial-defined fields are still an open question. On the basis of Table 1 and further experimental results, we found further evidence for an observation of Ahmadi and Menezes [2]: if the degree $d \equiv \pm 3(\bmod 8)$, the degree of the sediment is at least $d / 3$, whereas if $d \equiv \pm 1(\bmod 8)$, then the degree of the sediment is usually quite small.

For the extension degrees for which there are no trinomials we computed not only the square root friendly pentanomials but also the heptanomials with smallest degree sediment - the idea was, that perhaps one can find good heptanomials with a lower degree sediment than the best pentanomials, to improve modular reduction 2: they are given in Table 2 Similar searches for polynomials with nine and eleven terms have been performed. We immediately observe here that sediment degree differences are very limited, so the heptanomials do not bring advantages. The same observation applies to polynomials with nine or eleven terms. A pattern in the distribution of degrees of the second term of the sediment of square root friendly polynomials up to degree 3000 and with up to eleven terms prompts us to formulate the following conjecture:

Conjecture 1. Let $d$ be an odd positive integer, and let $c$, resp. $c^{\prime}$ be the exponents of the second, resp. third largest exponent in the lexicographically minimal square root friendly polynomial of degree $d$. Then

$$
3 c-d=c-c^{\prime}=\left\{\begin{array}{lll}
8 & \text { if } d \equiv 1 & (\bmod 3) \\
4 & \text { if } d \equiv 2 & (\bmod 3)
\end{array} .\right.
$$

A first result in this direction has already been proved by Bluher [16] using a result of Swan [29] (that in fact goes back to Stickelberger). Her result is: The odd degree polynomial $p(X)=X^{d}+\sum_{i \in \mathcal{S}} X^{i}+1$ in $\mathbb{F}_{2}[X]$, where $\mathcal{S} \subset\{i$ : $i$ odd, $0<i<d / 3\} \cup\{i: i \equiv d(\bmod 4), 0<i<d\}$ has no repeated roots; if $d= \pm 1(\bmod 8)$, then $f$ has an odd number of irreducible factors; and if $d= \pm 3$ (mod 8), then $f$ has an even number of irreducible factors. In fact, by adapting the proof given in [3] of Bluher's result, it is possible to prove the following theorem:

Theorem 3. Let $d$ be an odd positive integer and the polynomial $p(X) \in \mathbb{F}_{2}[x]$ have degree $d$ and satisfy one of the following conditions:

1. If $d \equiv 1(\bmod 3)$, then $p(X)$ is either of the form

$$
\begin{aligned}
& -X^{d}+X^{\frac{d+8}{3}}+\sum_{j \in J} X^{j}+1 \text { where } J=\left\{j: j \text { odd, } 1 \leq j<\frac{d-19}{3}\right\} \\
& \text { - or } X^{d}+\sum_{j \in J^{\prime}} X^{j}+1 \text { where } J^{\prime}=\left\{j: j \text { odd, } 1 \leq j \leq \frac{d+5}{3}\right\}
\end{aligned}
$$

2. If $d \equiv 2(\bmod 3)$, then $p(X)$ is either of the form

\footnotetext{
${ }^{2}$ This quite common assumption is heuristic and not necessarily always true [28. The search for polynomials with minimal degree sediment among those with given properties is however an interesting mathematical endeavor per se.
} 
Table 2. Some special square root friendly heptanomials

\begin{tabular}{c|l|l}
\hline \hline Degree & \multicolumn{1}{|c|}{ Irreducible heptanomial } & \multicolumn{1}{c}{$\zeta=\sqrt{x}$} \\
\hline 53 & $X^{53}+X^{19}+X^{15}+X^{5}+X^{3}+X+1$ & $x^{27}+x^{10}+x^{8}+x^{3}+x^{2}+x$ \\
59 & $X^{59}+X^{21}+X^{17}+X^{13}+X^{3}+X+1$ & $x^{30}+x^{11}+x^{9}+x^{7}+x^{2}+x$ \\
67 & $X^{67}+X^{25}+X^{17}+X^{7}+X^{3}+X+1$ & $x^{34}+x^{13}+x^{9}+x^{4}+x^{2}+x$ \\
83 & $X^{83}+X^{29}+X^{25}+X^{7}+X^{5}+X^{3}+1$ & $x^{42}+x^{15}+x^{13}+x^{4}+x^{3}+x^{2}$ \\
101 & $X^{101}+X^{35}+X^{31}+X^{9}+X^{7}+X+1$ & $x^{51}+x^{18}+x^{16}+x^{5}+x^{4}+x$ \\
107 & $X^{107}+X^{37}+X^{33}+X^{15}+X^{9}+X^{7}+1$ & $x^{54}+x^{19}+x^{17}+x^{8}+x^{5}+x^{4}$ \\
109 & $X^{109}+X^{39}+X^{31}+X^{9}+X^{5}+X^{3}+1$ & $x^{55}+x^{20}+x^{16}+x^{5}+x^{3}+x^{2}$ \\
131 & $X^{131}+X^{45}+X^{41}+X^{13}+X^{9}+X+1$ & $x^{66}+x^{23}+x^{21}+x^{7}+x^{5}+x$ \\
139 & $X^{139}+X^{49}+X^{41}+X^{7}+X^{5}+X^{3}+1$ & $x^{70}+x^{25}+x^{21}+x^{4}+x^{3}+x^{2}$ \\
149 & $X^{149}+X^{51}+X^{47}+X^{9}+X^{7}+X+1$ & $x^{75}+x^{26}+x^{24}+x^{5}+x^{4}+x$ \\
157 & $X^{157}+X^{55}+X^{47}+X^{15}+X^{9}+X^{3}+1$ & $x^{79}+x^{28}+x^{24}+x^{8}+x^{5}+x^{2}$ \\
163 & $X^{163}+X^{57}+X^{49}+X^{15}+X^{9}+X+1$ & $x^{82}+x^{29}+x^{25}+x^{8}+x^{5}+x$ \\
179 & $X^{179}+X^{61}+X^{57}+X^{13}+X^{9}+X^{5}+1$ & $x^{90}+x^{31}+x^{29}+x^{7}+x^{5}+x^{3}$ \\
211 & $X^{211}+X^{73}+X^{65}+X^{13}+X^{11}+X^{3}+1$ & $x^{106}+x^{37}+x^{33}+x^{7}+x^{6}+x^{2}$ \\
251 & $X^{251}+X^{85}+X^{81}+X^{7}+X^{5}+X^{3}+1$ & $x^{126}+x^{43}+x^{41}+x^{4}+x^{3}+x^{2}$ \\
269 & $X^{269}+X^{91}+X^{87}+X^{15}+X^{13}+X^{11}+1$ & $x^{135}+x^{46}+x^{44}+x^{8}+x^{7}+x^{6}$ \\
283 & $X^{283}+X^{97}+X^{89}+X^{13}+X^{9}+X+1$ & $x^{142}+x^{49}+x^{45}+x^{7}+x^{5}+x$ \\
571 & $X^{571}+X^{193}+X^{185}+X^{15}+X^{11}+X^{3}+1$ & $x^{286}+x^{97}+x^{93}+x^{8}+x^{6}+x^{2}$ \\
\hline
\end{tabular}

$$
\begin{aligned}
& -X^{d}+X^{\frac{d+4}{3}}+\sum_{j \in J} X^{j}+1 \text { where } J=\left\{j: j \text { odd, } 1 \leq j \leq \frac{d-11}{3}\right\} \\
& \text { - or } X^{d}+\sum_{j \in J^{\prime}} X^{j}+1 \text { where } J^{\prime}=\left\{j: j \text { odd, } 1 \leq j \leq \frac{d+1}{3}\right\} .
\end{aligned}
$$

Then $p(X)$ is square-free. Furthermore, if $d \equiv \pm 1 \bmod 8$ then $p(X)$ has an odd number of irreducible factors, whereas $d \equiv \pm 3 \bmod 8$ then $p(X)$ has an even number of irreducible factors and therefore must be reducible.

Remark 5. We already mentioned in Remark 4 that in [2] the polynomials we study are proven to permit efficient computation of traces. In Conjecture 9 of [2] it is also speculated that if an irreducible pentanomial of degree $d$ exists, then an irreducible pentanomial of the same degree defining a polynomial basis with only one trace-one element also exists. In the similar vein we could try to restrict our Conjecture 1 to pentanomials, but we have the following example for degree 1987:

$$
X^{1987}+X^{665}+X^{661}+X^{549}+1 .
$$

This polynomial has minimal degree sediment, and the sediment has minimal degree second term among all irreducible pentanomials with minimal degree sediment. We have $d \equiv 1(\bmod 3)$ and $3 c-d=8$, but the second term of the sediment has degree 661 , not 657 . On the other hand, with heptanomials we find following irreducible

$$
X^{1987}+X^{665}+X^{657}+X^{25}+X^{21}+X^{9}+1
$$

and we know of no other irreducibles of the form 


$$
X^{1987}+X^{c}+X^{c^{\prime}}+\text { other lower odd degree terms }+1
$$

with $c$ smaller than 665 or with $c=665$ and $c^{\prime}$ smaller than 657 .

\section{Implementation}

For several binary fields $\mathbb{F}_{2^{d}}$ we implemented a few operations, including (but not only) multiplication, squaring, square roots, and also trace and half-trace computations (the latter is used for solving quadratic equations). The current implementation is an extension of the implementation described in [1], that is also used in 12 and [6], (some performance discrepances are due to the fact that some other routines have been improved in the meantime) and as such in the current version processes the operands with a 32-bit granularity.

Depending on the nature of the chosen reduction polynomial we implemented some routines such as modular reduction and square root extraction in different ways. In a few cases we implement the same field twice using two different reduction polynomials in order to compare the different situations.

\subsection{Extracting a Square Root}

To implement square root extraction of a field element $\alpha$ we use formula (1): the only part that changes between different implementations is how the multiplication $\zeta \cdot \alpha_{\text {odd }}$ is realized.

To compute $\alpha_{\text {even }}$ and $\alpha_{\text {odd }}$ from $\alpha$ we use a 16-bit to 8 -bit look-up table to compress the bits. Using a trick due to Robert Harley, this table is stored in 256 consecutive bytes by mapping the input $i$ to $(i+i \gg 7) \& 0 x f f$.

If the reduction polynomial $p(X)$ is not square root friendly, then the Hamming weight of the square root is counted at the time of initialization of the library - hence only once. If this weight is low enough then a simple routine that XORs together shifted copies of $\alpha_{\text {odd }}$ according to which bits in $\zeta$ are set is used. If the weight is higher than a certain threshold, then a comb binary polynomial multiplication method is used, where the precomputations relative to $\zeta$ are performed only once, at initialization time. A reduction modulo $p(X)$ is then performed, if necessary.

By means of this we can always keep the time of the multiplication $\zeta \cdot \alpha_{\text {odd }}$ to just under a half of the time of a generic field multiplication: Note, however, that this time can be substantially higher than the time required for the per-field, ad-hoc optimized implementation of multiplication, as our experimental results in $\$ 5.4$ show.

In the case where the degree $d$ reduction polynomial $p(X)$ is square root friendly, we also have a simple routine that XORs together shifted copies of $\alpha_{\text {odd }}$ according to which bits in $\zeta$ are set, but since the weight is very low and known, the routine can be unrolled completely. Furthermore, as already mentioned, in this case no modular reduction is necessary. This routine is the one that delivers the best performance. 


\subsection{Trace Computation}

We follow here 20 and 20. For generic binary fields, to compute the trace from $\mathbb{F}_{2^{d}}$ to $\mathbb{F}_{2}$ we use the fact that it is linear. In other words, once we have computed $\operatorname{Tr}\left(x^{i}\right)$ for all $i$ with $0 \leq i<d$, in order to compute the trace of an element $\alpha \in \mathbb{F}_{2^{d}}, \alpha=\sum_{i=0}^{d-1} a_{i} x^{i}$ where the $a_{i} \in \mathbb{F}_{2}$, we have

$$
\operatorname{Tr}(\alpha)=\sum_{i=0}^{d-1} a_{i} \operatorname{Tr}\left(x^{i}\right) .
$$

The latter sum can be implemented by a componentwise multiplication (hence, a logical AND operation) of the bit vector representing the element $\alpha$ with a bit vector whose $i$-th component (starting with the zeroth component!) is the trace of $x^{i}$, called the trace vector, followed by a bit count modulo 2 . Both operations can be performed very efficiently. The trace vector is of course computed once for each field.

However, when a square root friendly polynomial is used to represent the field $\mathbb{F}_{d}$ with $d$ odd, the trace vector contains just one bit set, namely the least significant bit, and $\operatorname{Tr}(\alpha)=a_{0}$.

We therefore implemented two different routines: the first one uses a trace vector and the second one just polls the value of a single bit.

\subsection{Solving Quadratic Equations (Half-Traces)}

In order to solve quadratic equations of the form

$$
\lambda^{2}+\lambda=\alpha
$$

for $\lambda \in \mathbb{F}_{2^{d}}$ where $\alpha \in \mathbb{F}_{2^{d}}$ we implement just one generic routine, that takes the element $\alpha$ as an input.

The half-trace operator on $\mathbb{F}_{2^{d}}(d$ odd) is defined as

$$
H(\alpha)=\sum_{i=0}^{(d-1) / 2} \alpha^{2^{2 i}} .
$$

It is easily verified that it is $\mathbb{F}_{2}$-linear, and that it satisfies $H(\alpha)^{2}+H(\alpha)=$ $\alpha+\operatorname{Tr}(\alpha)$. Therefore, in order to solve equation (5) we first have to check whether $\operatorname{Tr}(\alpha)=0$. Only in that case (5) solvable: Then we compute the half-trace $H(\alpha)$ of $\alpha$ by adding together precomputed half-traces of elements of the polynomial base, i.e. $H\left(x^{i}\right)$, and $H(\alpha), H(\alpha)+1$ are the solutions.

One optimization in [20] consists in removing the coefficients of even powers of $x$ : We write $H(\alpha)=H\left(\alpha^{\prime}\right)+\beta$ where $\alpha^{\prime}$ has fewer nonzero coefficients than $\alpha$. This can be done by observing that $H\left(x^{2 i}\right)=H\left(x^{i}\right)+x^{2 i}+\operatorname{Tr}\left(x^{2 i}\right)$. Therefore each coefficient of an even power of $x$ can be set to zero. This halves the amount of stored half-traces of powers of $x$.

Our approach differs from the one in 20 in that we do not make ad-hoc attempts to minimize memory requirements. Instead, we reduce the number of 
half-traces to be accumulated by increasing the amount of precomputations. We compute and store $H\left(\ell_{0} x^{8 i+1}+\ell_{1} x^{8 i+3}+\ell_{2} x^{8 i+5}+\ell_{3} x^{8 i+7}\right)$ for all $i \geq 0$ such that $8 i+1 \leq d-2$ and all $\left(\ell_{0}, \ell_{1}, \ell_{2}, \ell_{3}\right) \in \mathbb{F}_{2}^{4} \backslash\{(0,0,0,0)\}$ such that the degree of the argument of $H$ is at most $d-2$. By means of this we reduce by a factor $32 / 15$ the expected number of table lookups and additions of half-traces.

\subsection{Results, Conclusions}

We benchmarked our implementation. Table 3 collects the timings of our routines on a PowerPC G4 running at $1.5 \mathrm{Ghz}$. (Table 5 shows the timings on an Intel Core 2 Duo CPU running at $1.83 \mathrm{Ghz}$.) Several field operations are timed, and the costs relative to a field multiplication are provided. The reduction polynomials are given (as decreasing lists of exponents) as well as whether the considered polynomial is square root friendly or not.

We have chosen degrees in various more or less evenly spaced ranges to fulfil the following requirements: the "classical" extension degrees 163, 191, and 233 must be included, fields around integer submultiples of these sizes should also be taken into account (that's the reason for degrees in the ranges 40-50 and 80100 , and 127), and we should provide examples of fields where the "standard" polynomial (either in the sense that it comes from standard documents, or that it is the most common one used for computations in computer algebra systems) already is square root friendly as well as cases where it is not - in the latter scenario we also choose a second defining polynomial that is square root friendly. Furthermore, all these combination of cases should happen with trinomial defined fields as well as when pentanomials are used. The "submultiples" ranges are relevant because of Trace Zero Varieties coming from elliptic curves [18], and

Table 3. Operations in some Binary Fields on a PowerPC G4 running at 1.5 Ghz. Mul, Sqr, Inv, Sqrt, Tr and Eq denote multiplication, squaring, field inversion, square root extraction, trace and half-trace computation respectively

\begin{tabular}{|c|c|c|c|c|c|c|c|c|c|c|c|c|c|}
\hline \multirow{2}{*}{$\begin{array}{l}\text { Field } \\
\text { (Bits) }\end{array}$} & \multirow{2}{*}{$\begin{array}{l}\text { Reduction } \\
\text { Polynomial }\end{array}$} & \multirow{2}{*}{$\begin{array}{l}\text { Sqrt } \\
\text { Frnd } \\
\end{array}$} & \multicolumn{6}{|c|}{ Operation Timings $(\mu$ sec $)$} & \multicolumn{5}{|c|}{ Costs relative to one Mul } \\
\hline & & & Mul & Sqr & Inv & Sqrt & Trace & Eq & Sqr & Inv & Sqrt & Trace & Eq \\
\hline$\overline{41}$ & $41,3,0$ & Yes & .084 & .013 & .448 & .015 & .007 & .129 & .160 & 5.340 & .178 & .078 & 1.536 \\
\hline 43 & $43,6,4,3,0$ & No & .090 & .016 & .453 & .327 & .020 & .128 & .179 & 5.010 & 3.619 & .227 & 1.422 \\
\hline 43 & 43,17 & Yes & .090 & .017 & .454 & .018 & .007 & .126 & .186 & 5.029 & .196 & .072 & 1.400 \\
\hline 47 & $47,5,0$ & Yes & .089 & .013 & .478 & .015 & .007 & .128 & .150 & 5.344 & .167 & .083 & 1.438 \\
\hline 83 & 83,7, & No & .212 & .030 & .915 & .383 & .023 & .191 & .141 & 4.313 & 1.806 & .107 & .901 \\
\hline 83 & $83,29,25,3,0$ & Yes & .214 & .045 & .925 & .054 & .007 & .192 & .209 & 4.316 & .254 & .034 & .897 \\
\hline 89 & 89,3 & No & .253 & .023 & .958 & .354 & .023 & .200 & .089 & 3.782 & 1.398 & .092 & .791 \\
\hline 89 & 8 & Yes & .253 & .024 & .958 & .025 & .007 & .201 & .093 & 3.782 & .098 & .027 & .794 \\
\hline 97 & 97,6 & No & .311 & .028 & 1.598 & .401 & .025 & .256 & .091 & 5.139 & 1.289 & .081 & .823 \\
\hline 97 & $97,33,0$ & Yes & .304 & .024 & 1.576 & .028 & .006 & .252 & .080 & 5.191 & .091 & .020 & .829 \\
\hline 127 & $127,1,0$ & Yes & .418 & .053 & 1.814 & .062 & .007 & .288 & .127 & 4.345 & .149 & .016 & .689 \\
\hline 163 & $163,7,6$ & No & .819 & .086 & 5.317 & .679 & .033 & .388 & .105 & 6.491 & .829 & .040 & .474 \\
\hline 163 & $163,57,49,29,0$ & Yes & .821 & .090 & 5.327 & .095 & .007 & .391 & .110 & 6.491 & .116 & .009 & .476 \\
\hline 191 & $\begin{array}{l}191,9,0 \\
\end{array}$ & Yes & .896 & .083 & 5.804 & .093 & .008 & .429 & .093 & 6.474 & .104 & .009 & .479 \\
\hline 223 & $223,33,0$ & Yes & 1.198 & .098 & 12.865 & .113 & .006 & .501 & .082 & 10.737 & .094 & .005 & .418 \\
\hline 233 & 233,7 & No & 1.336 & .101 & 14.599 & .918 & .037 & .582 & .076 & 10.925 & .687 & .028 & .436 \\
\hline 233 & $233,159,0$ & Yes & 1.330 & .090 & 14.593 & .117 & .006 & .584 & .068 & 10.971 & .088 & .005 & .439 \\
\hline
\end{tabular}


future investigation will consider the use of point and divisor halving for these algebraic groups.

Our implementation shows some interesting results.

1. The claims made in [20] about the speed of optimized square root extraction for fields defined by suitable trinomials are extended to pentanomials. Our results show for fields of 163, 191, and 233 bits an even further reduced Sqrt/Mul ratio. The gain w.r.t. generic implementations of square roots ranges from 8 to 20, and it is higher for smaller fields.

2. Multiplication and squaring may get marginally slower because the square root friendly polynomials usually do not have minimal degree sediments, and thus the reduction routine has to be a bit longer. The differences are minimal, field squaring paying a slightly higher toll than field multiplication. Sometimes a negligible performance improvement (presumably due to randomness) is observed. Field inversion and half-trace computation are unaffected by the choice of polynomial.

3. Computing traces with square root friendly polynomials takes nearly negligible time.

4. In [20] computing an half-trace requires approximately $2 / 3$ the time of a field multiplication, but for fields of the same sizes our ratios are lower than $1 / 2$, because we use a lot of precomputations.

In Table 4 we give estimates of the costs relative to one field multiplication of elliptic curve group addition, doubling, halving and scalar multiplication using various algorithms. We have used the operations counts from [5, $\S 5.2]$, but the ratios between field operations come from our Table 3. We can see that the use square root friendly polynomials has a noticeable impact on scalar multiplication performance based on point halving. Point halving alone is sped up by about $20 \%$, and the whole scalar multiplication by about $14 \%$ for curves defined over $\mathbb{F}_{2^{163}}$ and by $11 \%$ if the base field is $\mathbb{F}_{2^{233}}$. These improvements are much larger than the difference in field multiplication performance (a $2.5 \%$ loss for $\mathbb{F}_{2^{163}}$,

Table 4. Estimated costs of elliptic curve group operations and scalar multiplication relative to those of a field multiplication. Add, resp. Dbl, Hlv mean Addition, resp. Doubling, Halving, and D \& A, resp. H \& A means windowed scalar multiplication based on Double-and-Add, resp. Halve-and-Add. Scalar multiplications methods use affine coordinates as well as mixed affine-Lopez-Dahab coordinates.

\begin{tabular}{c|c||r|r|r|r||r|r|r}
\hline \hline \multicolumn{2}{c||}{ Field } & \multicolumn{4}{c||}{ Group Operations } & \multicolumn{3}{c}{ Scalar Multiplication } \\
\hline \multirow{2}{*}{ Degree } & Sqrt & Affine Coords. & \multicolumn{2}{c|}{ Mixed $\mathcal{A}+\mathcal{L D}$} & \multicolumn{2}{|c}{ Affine Coords. } & Mixed \\
\cline { 3 - 8 } & Frnd & Add,Dbl & Hlv & mixed A & Dbl & D \& A & H\& A & D \&A \\
\hline \hline 163 & $\mathrm{~N}$ & 8.596 & 3.343 & 8.525 & 5.175 & 1550.3 & 834.5 & 1137.2 \\
163 & $\mathrm{Y}$ & 8.601 & 2.601 & 8.550 & 5.200 & 1551.4 & 715.0 & 1141.1 \\
\hline 191 & $\mathrm{Y}$ & 8.567 & 2.592 & 8.465 & 5.115 & 1801.3 & 825.0 & 1310.7 \\
\hline 233 & $\mathrm{~N}$ & 13.001 & 3.151 & 8.380 & 5.030 & 3369.4 & 1291.0 & 1588.6 \\
233 & $\mathrm{Y}$ & 13.039 & 2.532 & 8.340 & 4.990 & 3379.2 & 1149.4 & 1579.8 \\
\hline
\end{tabular}


Table 5. Operations in some Binary Fields on an Intel Core 2 Duo running at $1.83 \mathrm{Ghz}$

\begin{tabular}{|c|c|c|c|c|c|c|c|c|c|c|c|c|c|}
\hline \multirow{2}{*}{$\begin{array}{l}\text { Field } \\
\text { (Bits) }\end{array}$} & \multirow{2}{*}{$\begin{array}{l}\text { Reduction } \\
\text { Polynomial }\end{array}$} & \multirow{2}{*}{$\begin{array}{l}\text { Sqrt } \\
\text { Frnd }\end{array}$} & \multicolumn{6}{|c|}{ Operation Timings $(\mu \mathrm{sec})$} & \multicolumn{5}{|c|}{ Costs relative to one Mul } \\
\hline & & & Mul & Sqr & Inv & Sqrt & Trace & $\mathrm{Eq}$ & Sqr & Inv & Sqrt & \begin{tabular}{|l|} 
Trace \\
\end{tabular} & $\mathrm{Eq}$ \\
\hline 41 & $41,3,0$ & Yes & .065 & .019 & 1.343 & .023 & .012 & .057 & .292 & 20.662 & .358 & .184 & .883 \\
\hline 43 & $43,6,4,3,0$ & No & .067 & .017 & 1.381 & .141 & .023 & .057 & .253 & 20.611 & 2.099 & .337 & .837 \\
\hline 43 & $43,17,9$ & Yes & .069 & .021 & 1.368 & .021 & .012 & .057 & .304 & 19.826 & .326 & .174 & .829 \\
\hline 47 & $47,5,0$ & Yes & .074 & .015 & 1.452 & .025 & .012 & .063 & .203 & 19.621 & .335 & .162 & .853 \\
\hline 83 & $83,7,4,2,0$ & No & .184 & .041 & 2.727 & .245 & .022 & .094 & .223 & 14.821 & 1.333 & .120 & .512 \\
\hline 83 & $83,29,25,3,0$ & Yes & .172 & .035 & 2.727 & .049 & .012 & .092 & .203 & 15.855 & .285 & .070 & .537 \\
\hline 89 & $89,38,0$ & No & .207 & .028 & 2.813 & .203 & .018 & .083 & .135 & 13.589 & .982 & .089 & .402 \\
\hline 89 & $89,51,0$ & Yes & .201 & .036 & 2.837 & .044 & .012 & .080 & .179 & 14.114 & .217 & .060 & .394 \\
\hline 97 & $97,6,0$ & No & .223 & .032 & 3.677 & .200 & .021 & .118 & .144 & 16.523 & .898 & .093 & .530 \\
\hline 97 & $97,33,0$ & Yes & .220 & .031 & 3.670 & .046 & .012 & .118 & .141 & 16.489 & .201 & .054 & .536 \\
\hline 127 & $127,1,0$ & Yes & .305 & .038 & 4.375 & .050 & .012 & .122 & .125 & 14.344 & .164 & .039 & .400 \\
\hline 163 & $163,7,6,3,0$ & No & .495 & .069 & 6.855 & .314 & .028 & .160 & .139 & 13.855 & .635 & .056 & .324 \\
\hline 163 & $163,57,49,29,0$ & Yes & .445 & .078 & 7.055 & .066 & .012 & .158 & .175 & 13.848 & .148 & .027 & .355 \\
\hline 191 & $191,9,0$ & Yes & .648 & .051 & 8.873 & .052 & .012 & .243 & .079 & 13.692 & .081 & .018 & .375 \\
\hline 223 & $223,33,0$ & Yes & .775 & .072 & 9.413 & .073 & .012 & .313 & .093 & 12.415 & .094 & .015 & .404 \\
\hline 233 & 233,74 & No & .945 & .086 & 12.246 & .358 & .019 & .321 & .091 & 12.959 & .379 & .020 & .340 \\
\hline 233 & $233,159,0$ & Yes & .951 & .094 & 12.225 & .070 & .012 & .320 & .099 & 12.854 & .074 & .013 & .336 \\
\hline
\end{tabular}

a $4.5 \%$ gain for $\left.\mathbb{F}_{2^{233}}\right)$. It is safe to assume that similar improvements can be achieved on the Yao-like scalar multiplication algorithms from [5].

We already mentioned that square root extractions are used in some pairing computation algorithms. For instance, the main loop of Algorithm 2 from 13 requires 7 multiplication, two squarings and two square root extractions in fields of even characteristic. In this context, the overall gain obtained by faster square root extraction is going to be less important than with point halving, but still noticeable.

Square root friendly polynomials should be used when implementing formula that make heavy use of square root extraction in fields of characteristic two. For solving quadratic equations then we advise to increase the amount of precomputed half-traces to improve performance. In particular, the improvements in scalar multiplication performance based on point halving obtained in [20] by using special trinomial can be carried over to fields defined by pentanomials. Around $20 \%$ can be expected for the point halving alone, with an impact of $11 \%$ to $14 \%$ on the entire scalar multiplication.

Acknowledgement. The author acknowledges interesting discussions on the subject with Peter Birkner, Toni Bluher, Darrel Hankerson, Alfred Menezes, Mike Scott, and Nicolas Thériault.

\section{References}

1. Ahmadi, O., Hankerson, D., Menezes, A.: Formulas for cube roots in $\mathbb{F}_{3 m}$. Discrete Applied Math. 155(3), 260-270 (2007)

2. Ahmadi, O., Menezes, A.: On the number of trace-one elements in polynomial bases for $\mathbb{F}_{2^{n}}$. Designs, Codes and Cryptography 37, 493-507 (2005) 
3. Ahmadi, O., Menezes, A.: Irreducible polynomials of maximum weight. Utilitas Mathematica 72, 111-123 (2007)

4. Ash, D.W., Blake, I.F., Vanstone, S.: Low complexity normal bases. Discrete Applied Math. 25, 191-210 (1989)

5. Avanzi, R.M.: Delaying and Merging Operations in Scalar Multiplication: Applications to Curve-Based Cryptosystems. In: Proceedings of SAC 2006 (to appear)

6. Avanzi, R.M., Cesena, E.: Trace Zero Varieties over Fields of Characteristic 2: Cryptographic Applications. In: SAGA 2007. The first Symposium on Algebraic Geometry and its Applications, May 7-11, 2007,Tahiti (2007)

7. Avanzi, R.M., Ciet, M., Sica, F.: Faster Scalar Multiplication on Koblitz Curves combining Point Halving with the Frobenius Endomorphism. In: Bao, F., Deng, R., Zhou, J. (eds.) PKC 2004. LNCS, vol. 2947, pp. 28-40. Springer, Heidelberg (2004)

8. Avanzi, R., Cohen, H., Doche, C., Frey, G., Lange, T., Nguyen, K., Vercauteren, F.: The Handbook of Elliptic and Hyperelliptic Curve Cryptography. CRC Press, Boca Raton, USA (2005)

9. Avanzi, R.M., Heuberger, C., Prodinger, H.: Scalar Multiplication on Koblitz Curves Using the Frobenius Endomorphism and its Combination with Point Halving: Extensions and Mathematical Analysis. Algorithmica 46, 249-270 (2006)

10. Avanzi, R.M., Heuberger, C., Prodinger, H.: On Redundant $\tau$-adic Expansions and Non-Adjacent Digit Sets. In: Biham, E., Youssef, A.M. (eds.) SAC 2006. LNCS, vol. 4356, Springer, Heidelberg (2006)

11. Avanzi, R.M., Thériault, N.: Effects of Optimizations for Software Implementations of Small Binary Field Arithmetic. In: Proceedings of WAIFI 2007. International Workshop on the Arithmetic of Finite Fields, June 21-22, 2007, Madrid, Spain, p. $18(2007)$

12. Avanzi, R.M., Thériault, N., Wang, Z.: Rethinking Low Genus Hyperelliptic Jacobian Arithmetic over Binary Fields: Interplay of Field Arithmetic and Explicit Formulæ. CACR Technical Report 2006-07

13. Barreto, P.S.L.M., Galbraith, S., OhEigeartaigh, C., Scott, M.: Efficient pairing computation on supersingular abelian varieties. Designs, Codes and Cryptography 42, 239-271 (2007), http://eprint.iacr.org/2004/375

14. Birkner, P.: Efficient Divisor Class Halving on Genus Two Curves. In: Proceedings of Selected Areas in Cryptography - SAC 2006. LNCS, Springer, Heidelberg (to appear)

15. Birkner, P., Thériault, N.: Efficient Divisor Class Doubling and Halving on Genus Three Curves (in preparation)

16. Bluher, A.W.: A Swan-like Theorem. Finite Fields and Their Applications 12, 128 138 (2006)

17. Bosma, W., Cannon, J., Playoust, C.: The Magma Algebra System I: The User Language. J. Symbolic Comput. 24, 235-265 (1997)

18. Cesena, E.: Varietá a Traccia Zero su Campi Binari: Applicazioni Crittografiche (Trace Zero Varieties over Binary Fields: Cryptographic Applications.) Master's Thesis. Universitá degli Studi di Milano (in Italian) (2005)

19. Coron, J.-S., M'Raïhi, D., Tymen, C.: Fast generation of pairs $(k,[k] P)$ for Koblitz elliptic curves. In: Vaudenay, S., Youssef, A.M. (eds.) SAC 2001. LNCS, vol. 2259, pp. 151-164. Springer, Heidelberg (2001)

20. Fong, K., Hankerson, D., López, J., Menezes, A.: Field Inversion and Point Halving Revisited. IEEE Trans. Computers 53(8), 1047-1059 (2004)

21. Hankerson, D., Menezes, A.J., Vanstone, S.A.: Guide to elliptic curve cryptography. Springer, Heidelberg (2003) 
22. Knudsen, E.W.: Elliptic Scalar Multiplication Using Point Halving. In: Lam, K.-Y., Okamoto, E., Xing, C. (eds.) ASIACRYPT 1999. LNCS, vol. 1716, pp. 135-149. Springer, Heidelberg (1999)

23. Koblitz, N.: CM-curves with good cryptographic properties. In: Feigenbaum, J. (ed.) CRYPTO 1991. LNCS, vol. 576, pp. 279-287. Springer, Heidelberg (1992)

24. Lange, T., Stevens, M.: Efficient doubling for genus two curves over binary fields. In: Handschuh, H., Hasan, M.A. (eds.) SAC 2004. LNCS, vol. 3357, pp. 170-181. Springer, Heidelberg (2004)

25. National Institute of Standards and Technology. Recommended Elliptic Curves for Federal Government Use. NIST Special Publication (July 1999), available from: http://csrc.nist.gov/csrc/fedstandards.html

26. Schroeppel, R.: Point halving wins big. In: (i) Midwest Arithmetical Geometry in Cryptography Workshop, November 17-19, 2000, University of Illinois at UrbanaChampaign (2000), ECC 2001 Workshop, October 29-31, 2001, University of Waterloo, Ontario, Canada (2001)

27. Schroeppel, R.: Elliptic curve point ambiguity resolution apparatus and method. International Application Number PCT/US00/31014, filed (November 9, 2000)

28. Scott, M.: Optimal Irreducible Polynomials for $\mathrm{GF}\left(2^{m}\right)$ Arithmetic. IACR ePrint 2007/192. http://eprint.iacr.org/2007/192

29. Swan, R.G.: Factorization of Polynomials over Finite Fields. Pac. J. Math. 19, 1099-1106 (1962)

\section{A Comparing Some Modular Reduction Routines}

The use of a square root friendly polynomial can slow down modular reduction, but we already observed that this performance loss is minimal. This is explained by the fact that even though reduction does become more expensive, the amount of additional operations is rather small.

As an example, we report here the reduction code for the two degree 163 polynomials which we used. The input is given as eleven 32 -bit words $r A, r 9, r 8, \ldots$, $\mathrm{r} 1, \mathrm{r} 0$ and the reduced output is computed in place in the six least significant words $r 5, r 4, r 3, r 2, r 1, r 0$.

To reduce modulo $X^{163}+X^{7}+X^{6}+X^{3}+1$, the number of necessary logical operations between CPU registers is 74 . Reduction modulo $X^{163}+X^{57}+X^{49}+$ $X^{29}+1$ takes 89 logical operations. This example is the one with the largest complexity increase in all the comparisons we worked out.

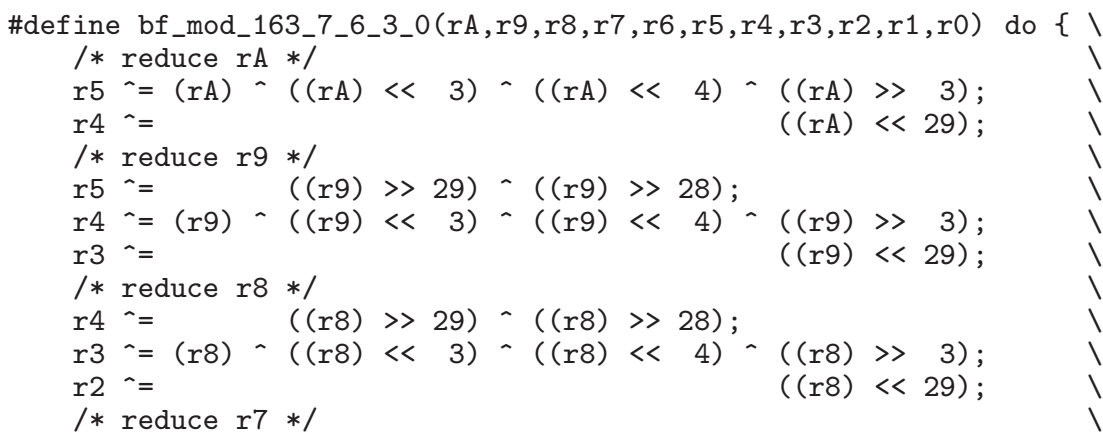



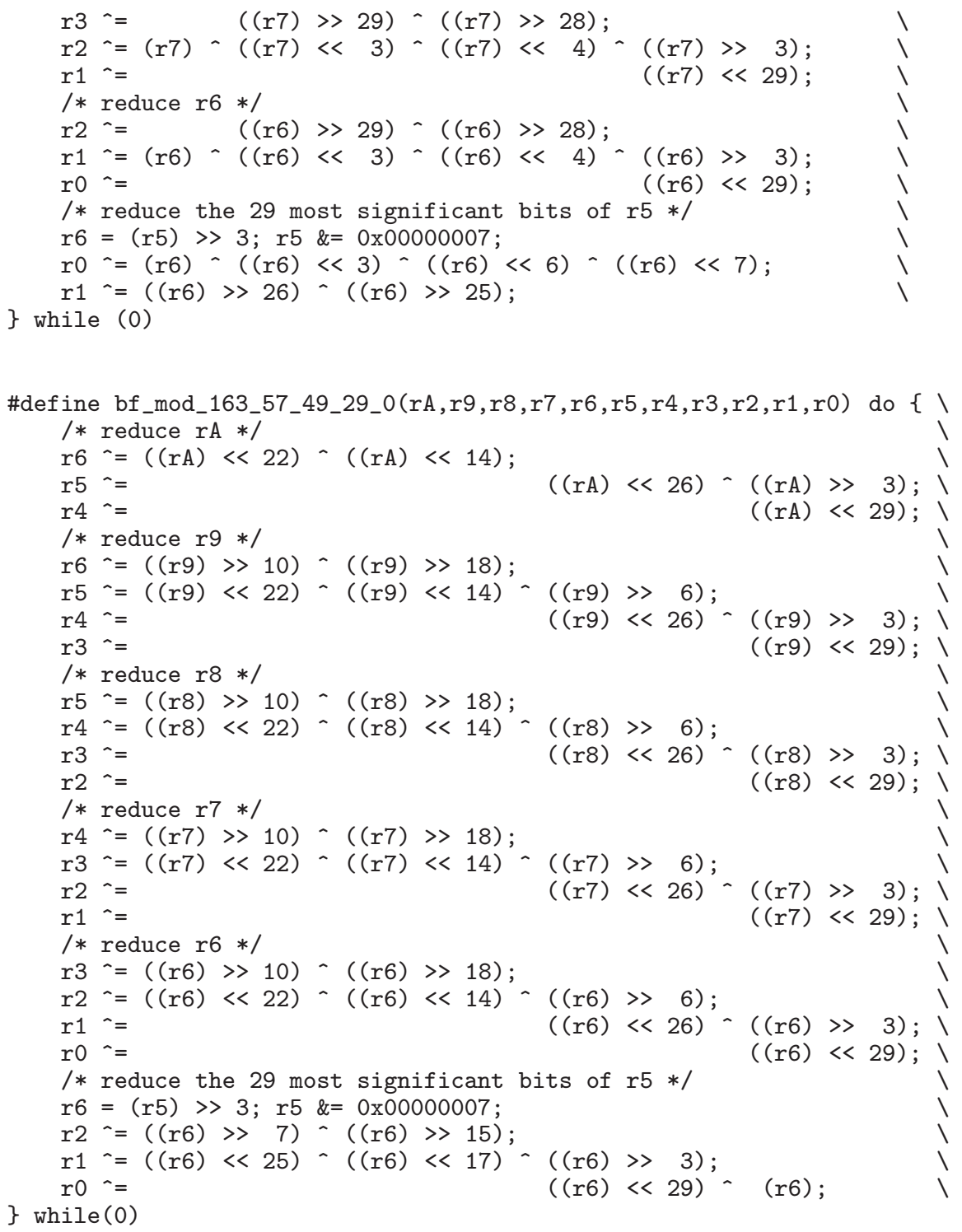